\title{
Cranberry SSR multiplexing panels for DNA horticultural fingerprinting and genetic studies
}

Brandon Schlautman ${ }^{\mathrm{a},}$, , Jenny Bolivar-Medina ${ }^{\mathrm{b}}$, Sarah Hodapp ${ }^{\mathrm{b}}$, Juan Zalapa ${ }^{\mathrm{b}, \mathrm{c}, *}$

a The Land Institute, 2440 E. Water Well Rd., Salina, KS 67401, USA.

b Department of Horticulture, University of Wisconsin-Madison, Madison, WI, USA.

c Vegetable Crops Research Unit, USDA-ARS, University of Wisconsin-Madison, Madison, WI, USA.

*Corresponding authors: B. Schlautman, schlautman@landinstitute.org; J. Zalapa, jezalapa@wisc.edu

\begin{abstract}
Cranberry (Vaccinium macrocarpon) is in need of inexpensive high-throughput DNA fingerprinting methods for genetic research and germplasm purity testing for agricultural purposes. Therefore, we designed and validated 16multiplexing panels containing 61 evenly distributed simple sequence (SSR) markers, with non-overlapping allele ranges, throughout the 12 cranberry linkage groups. Several important cranberry cultivars and selections $(\mathrm{n}=18)$ and a diploid accession of $V$. oxycoccos were genotyped with the multiplexing panels and separated through principal component analysis (PCA) to demonstrate their effectiveness for DNA fingerprinting and genetic diversity analysis. A subset of three multiplexing panels containing 12 SSR markers was used to genotype 174 seedlings from fruits collected in a commercial cranberry bed of the cultivar Stevens, and identification of intra-cultivar heterogeneity was investigated in the bed to validate the use of the markers in such future applications. Furthermore, determining the likelihood that each seedling was self- or cross-pollinated provided the first quantitative evidence $(p<0.0001)$ that the majority of seeds within commercial cranberry beds are self-pollinated. These multiplexing panels represent an important, applicable resource for cranberry researchers and farmers of the North American industry. These markers can be used to assess the genetic homogeneity of grower and licensed propagators' cranberry beds, to protect the intellectual property rights of plant breeders, and to enable cranberry researchers to monitor the genetic identity of genotypes within their breeding programs and genetic studies.
\end{abstract}

\section{Key words}

Vaccinium macrocarpon; Microsatellites; Multiplex-PCR; Genetic diversity; Clonal purity; Selfing

(C) 2017. This manuscript version is made available under the Elsevier user license http://www.elsevier.com/open-access/userlicense/1.0/ 


\section{Introduction}

The American cranberry (Vacinium macrocarpon Ait.) is a low trailing, woody shrub native to North America. It reproduces both sexually through flowers on short vertical branches and asexually through prolific, easily rooting stolons, which allow individual genotypes to form dense mats of vines covering multiple hectares in commercial cranberry beds (Brown and McNeil, 2006). During cranberry domestication in the mid-1800s, growers selected high yielding genotypes from wild stands based on fruit characteristics, and then propagated them as vegetative cuttings in large beds of a presumed single genotype (Peltier, 1970; Fajardo et al., 2012). This process was facilitated by several wild genotypes with unique berry architectural types (e.g., pyriform shaped Ben Lear). A collaborative culture of sharing and selling vines among cranberry producers quickly developed, and more than 100 such wild selections were made, named, clonally preserved, and sold in commercial production during the 19 th century (Dana et al., 1983). Today, cranberry

remains an asexually propagated crop, and producers still grow, share, and sell vines of some of the same wild selections from the $19^{\text {th }}$ century in addition to newer hybrid cultivars.

Cranberry beds can remain productive for many decades and gradually decrease in genetic homogeneity due to the establishment of selfed or outcrossed seedlings within the bed (Fajardo et al., 2012). Because vine and foliage characteristics vary little between cranberry genotypes (Chandler and Demoranville, 1958), unnoticed genetic diversity within cranberry beds whose vines are mowed, sold, or replanted in combination with human errors in record keeping has resulted in intra-clonal genetic heterogeneity within named wild selections and hybrid cultivars developed during the mid-20th century. Multiple studies have been performed to assess the extent of cultivar misclassification and genetic contamination of cranberry germplasm both in breeding programs and the industry in general using isozymes, random amplified polymorphic DNA (RAPD), sequence characterized amplified regions (SCAR), and simple sequence repeat (SSR) markers (Fajardo et al., 2012; Novy and Vorsa, 1995; Novy et al., 1996; Polashock and Vorsa, 2002; Boches, 2005). Furthermore, since the mid-2000s, a new generation of hybrid cultivars adapted to meet the needs of the modern cranberry farmers have been released (McCown and Zeldin, 2003; Clark and Finn, 2010). Several actions such as plant variety protection, use of licensed propagators for vine sales, and licensing agreements between breeders and growers have been undertaken in an attempt to maintain the genetic purity of these new cultivars. These actions and the royalties required to plant the new hybrid cultivars, combined with large financial input of preparing a cranberry bed to be planted have caused the replacement of existing beds to be a difficult decision and large investment for current grower's in the industry. 
Inexpensive and efficient high-throughput DNA fingerprinting technologies using available molecular markers should be an important additional strategy for maintaining the homogeneity of cranberry cultivars. Also, these same DNA fingerprinting technologies could serve as an affordable way for cranberry producers to determine the purity of aged beds when deciding to either replant the bed which has become genetically diverse or to adjust management practices in the bed which is still homogenous. In recent years, multiple studies have generated SSR markers from cranberry next generation sequence data (Schlautman et al., 2015a; Zhu et al., 2012; Georgi et al., 2011), and some of those SSRs have been positioned in genetic linkage maps (Georgi et al. 2013; Schlautman et al., 2015b). However, no standard high-throughput SSR fingerprinting multiplexing panels have been made available to cranberry researchers and industry.

The primary objective of this study was to develop cranberry high-throughput SSR multiplexing panels for inexpensive fingerprinting and testing of genetic diversity in cranberry germplasm. A total of 16 multiplexing panels containing 61 evenly distributed SSR markers, with non-overlapping allele ranges, throughout the 12 cranberry linkage groups were designed and validated in various multiplexing panels combinations to genotype a set of important wild and hybrid cranberry cultivars (Schlautman et al., 2015b). To demonstrate the discriminating power of the SSR multiplexing panels and to test the long-standing hypothesis that the majority of commercial cranberry fruits contain self-pollinated seeds, 3 selected panels, containing 12 SSR combinations, were used to determine whether seedlings from individual fruits collected from a commercial cranberry bed of the cultivar, Stevens, were self-pollinated or were pollinated by cultivars grown in surrounding beds.

\section{Materials and methods}

\subsection{SSR selection, grouping, and PCR conditions}

Using only markers with known genomic locations, the SSR primer-pairs were grouped into potential PCR multiplexing combinations of three or four SSRs using the following criteria: the SSRs must have non-overlapping allele ranges according to their previously published fragment length; they must not be linked, they must be located at greater than $10 \mathrm{cM}$ from the tip of each chromosome; they must anneal and amplify PCR products from a single genomic location; and they must not contain any known null alleles based on previous data (Schlautman et al., 2015a; Zhu et al., 2012; Georgi et al., 2011; Georgi et al. 2013; Schlautman et al., 2015b). Throughput potential was increased by pooling 4 PCR multiplex reactions, one for each dye (M13-FAM, HEX, NED, or PET labeled primer) and containing 3-4 SSR markers per dye to be analyzed in a single fragment analysis run. 
Forward primers from the selected SSRs were appended with the M13 sequence (5'-

CACGTTGTAAAACGAC-3') to allow for indirect fluorescent labeling of PCR products (Schuelke, 2000), and the PIG sequence (5'-GT'TTCT'T-3') was appended to reverse primers to promote full adenylation of SSR fragments during PCR (Brownstein et al., 1996). Using primers with the M13 sequence in the presence of a universal fluorescently labeled M13-tail is much more economic than direct fluorescent primer labeling (Guichoux et al., 1996); furthermore, it allows the primer-pairs and their multiplexed combinations to be indirectly labeled with any fluorescent type (color) suitable for the intended purpose rather than being limited to a specific dye.

Each multiplex PCR mixture was composed of $3.5 \mu$ 1 1xJumpstart RedTaq Ready Mix (Sigma, St. Louis, MO, USA), $1.0 \mu \mathrm{l}$ of $15 \mathrm{ng} / \mu \mathrm{l} \mathrm{DNA}, 1.5 \mu \mathrm{l}$ of $5 \mu \mathrm{M}$ forward primer, $1.5 \mu \mathrm{l}$ of $50 \mu \mathrm{M}$ reverse primer, and $0.5 \mu \mathrm{l}$ of $0.5 \mu \mathrm{M}$ M13-FAM, HEX, NED, or PET labeled primer. The $1.5 \mu \mathrm{l}$ of forward and reverse primer was divided by the number of multiplexed SSRs (i.e. $0.5 \mu \mathrm{l}$ and $0.375 \mu \mathrm{l}$ of forward and reverse primer from each SSR were added when 3 or 4 markers were multiplexed together, respectively). Thermocycling conditions were identical to those used in Schlautman et al. (2015a). Finally, $1 \mu \mathrm{l}$ of multiplexed PCR product from each of the four M13 dyes were pooled with $0.125 \mu$ l of LIZ500 standard ladder and $14.875 \mu \mathrm{l}$ formamide to create up to a 16x SSR poolplex. The poolplexed mixture was sent to the University of Wisconsin-Madison Biotechnology Center DNA sequencing facility for fragment analysis using an ABI 3730 fluorescent sequencer (Applied Biosystems, Foster City, CA, USA). Allele genotyping was performed using GeneMarker v2.63 (SoftGenetics LLC, State College, PA, USA).

It should be noted that multiplexing additives, often used to enhance multiplex reactions, were explicitly avoided in order to make DNA fingerprinting simpler and more economic for both cranberry growers and researchers. In addition, no attempt was made to adjust primer concentration during multiplex reactions. The main goal was to identify combinations of markers which could be multiplexed together under a universal protocol without having to use additional costly inputs or to reduce efficiency by using specific primer concentrations or thermocycling conditions for individual markers or marker combinations. Finally, PCR reactions were kept to a small volume $(8 \mu \mathrm{l})$ to further reduce associated costs, and the selected multiplex combinations worked sufficiently under these conditions. However, doubling each of the reactants to increase the reaction volume, and/or increasing the number of PCR cycles, can improve the quality of the resulting products in the form of more intense fluorescent peaks in the fsa files.

\subsection{Plant Materials}


A total of 18 unique cranberry ( $V$. macrocarpon) cultivars, consisting of wild selections from the east coast and from west of the Appalachian Mountains and of hybrids generated from crosses or backcrosses among wild cranberry selections, were genotyped in the initial screen of many different SSR multiplex combinations of three or four markers (Table 1). In addition, a diploid $V$. oxycoccos selection, a small-fruited cranberry species closely related to $V$. macrocarpon, was included to assess the transferability of these SSR marker combinations. Principal component analysis (PCA) was performed in $\mathrm{R}$ using the dudi.pca function in the ade4 package (Dray and Dufour, 2012). Genotype data for the 18 cranberry cultivars and the single $V$. oxycoccos accession was used to determine the number of alleles and allele ranges observed for each multiplexed marker.

In October 2014, 20 mature cranberry fruits were randomly selected from bulk fruit harvested from the center of a flooded commercial cranberry bed planted with the cultivar Stevens. The seeds of these fruits were extracted and 174 seeds ( $\sim 10$ seeds per fruit) were planted in the spring of 2016 after the seeds reached stratification requirements (Supplementary File 1). Cranberry is highly self-fertile, therefore, it was hypothesized that most of the seeds within those fruits would be pollinated by flowers within the same bed rather than outcrossed from a cultivar in the adjacent bed (Sarracino and Vorsa, 1991). To test this hypothesis, DNA was extracted from leaf tissue from each seedling, and 3 multiplex panels containing 12 loci were used to genotype the seedlings.

\subsection{Statistical analyses}

Determination of whether a seedling was self-pollinated or cross-pollinated was performed by classifying progeny as outcrossed when at least one or more alleles within one or more of the 12 loci could not have been inherited from Stevens. The progeny was assumed to be a self when all alleles at all loci were consistent with Stevens' inheritance. Additionally, principal component analysis (PCA) was performed in R using the dudi.pca function in the ade4 package, and individuals were colored within the plot of the first two principle components to visually assess whether progeny classified as selfs or outcross clustered together (Dray and Dufour, 2012). Finally, because some intra-cultivar genetic heterogeneity has previously been observed for multiple cranberry cultivars (Fajardo et al., 2012; Novy et al., 1996), we tested to see if some of the seeds may have arisen from fruits which were from some unknown cultivar rather than Stevens. We determined a seed to have a mother other than Stevens when it did not group with the other seedlings in the PCA, when it did not contain at least one allele from Stevens at every locus, or when one of its sibling seeds from the same fruit did not meet the above criteria. 
To test whether the number self-pollinated seeds was equal to the number of cross-pollinated seeds in the Stevens bed, chi-square tests were performed with $\mathrm{H}_{0}$ : proportion of self-pollinated seeds $=0.5$, proportion of crosspollinated seeds $=0.5$ and $\mathrm{H}_{1}: \mathrm{H}_{0}$ is false. Specifically, we performed $\chi^{2}=\sum \frac{(\text { observed \# of selfs-total seeds } \times 0.5)^{2}}{(\text { total seeds } \times 0.5)^{2}}$ with degrees of freedom equivalent to $k-1=1$ where $k$ is equal to the number of possible responses. In this case the possible responses were self-pollinated or cross-pollinated seedling and $k$ was 2 .

\section{Results and discussion}

\subsection{Effectiveness of SSR multiplexing panels for differentiating important cranberry cultivars and characterizing allelic diversity.}

A total of 16 different multiplexing panels containing three or four SSR markers which amplify well together in multiplex PCR reactions were identified (Table 2). These 16 multiplexing combinations fulfill the previously described selection and grouping requirements, and the 61 markers they contain span the entire cranberry genome with each of the twelve cranberry linkage groups represented by an average of 5.1 SSR loci (Table 2). A total of 388 alleles (an average of 6.4 alleles per locus) were observed across the 61 SSR markers for the 18 cranberry cultivars and the $V$. oxycoccos wild selection used. Because a universal M13 sequence was used for indirect fluorescent labeling, the multiplexing panels can

be used with any type of fluorescent label. Therefore, cranberry researchers and industry personnel can select subsets of multiplexing panels which contain the most heterozygous or informative markers for DNA fingerprinting of commercial beds or germplasm of interest.

$V$ oxycoccos had 27 private alleles not found in any of the cranberry cultivars, which was consistent with previous findings comparing genetic diversity of $V$. oxycoccos and $V$. macrocarpon (Zalapa et al., 2014). The crosstransferability of the markers and multiplexing panels suggests that they can be used to generate the first $V$. oxycoccos linkage map, which would facilitate the comparative genetic study between $V$. oxycoccos and $V$. macrocarpon. Comparative genetic studies between the two closely related species could promote future interspecific hybridization strategies for introgressing commercially important traits from V. oxycoccos into V. macrocarpon (Vorsa and Polashock, 2005).

PCA using the 61 markers clearly separated all 18 cranberry cultivars and the $V$. oxycoccos selection, even the closely related full-siblings HyRed and LoRed (Fig. 1). The first principal component (PC) explained 24.31\% of the total genetic variation; the second (PC) accounted for $19.79 \%$ of the variation. Although the sample size is small, some genetic structure and geographic relationships previously proposed in cranberry were observed (Schlautman et al., 2015a; Nilsen, 1995). Cranberry genotypes selected from east of the Appalachian Mountains tended to group in the bottom 
right quadrant, while genotypes selected from west of the Appalachian Mountains were located in the top left quadrant. Hybrids and backcrossed genotypes were located in-between as expected (Fig. 1). Interestingly, V. oxycoccos grouped near the $V$. macrocarpon genotypes selected from east of the Appalachian Mountains, suggesting that some migration could exist between native populations of the two species (Zalapa et al., 2014; Smith et al., 2015). The ability of these markers to distinguish between the cranberry genotypes demonstrates their potential relevance for cultivar discrimination for the protection of patented and released cranberry cultivars (Smith et al., 2015).

\subsection{Validation of multiplexing panel utility in a population of self-pollinated and cross-pollinated Stevens seedlings.}

Cranberry is capable of self-fertilization, therefore, it was hypothesized that the majority of seeds present within individual fruits and within an entire cranberry bed would arise mainly from self- rather than from cross-pollination events since beds are clonally propagated with a single cultivar (Sarracino and Vorsa, 1991). Previous studies have characterized the effect of self- and cross-pollination on the number of seeds per berry for a diverse number of cultivars (Sarracino and Vorsa, 1991; MacKenzie, 1995); however, studies characterizing the extent of self-pollination in a commercial cranberry bed have not been conducted.

DNA fingerprinting analysis using a subset of the SSR multiplexing combos designed herein was used to genotype the 174 seedlings from the 20 fruits collected in a Stevens commercial cranberry bed. A total of 146 of the 174 seedlings did not contain a single allele across the 12 loci amplified that was not inherited from Stevens, and these seedlings were considered to be results of self-pollination. The remaining 28 seedlings contained one or more allele which could not have been inherited from Stevens, and these seedlings were considered to be results of crosspollination. Interestingly, some of the cross-pollinated seedlings from one of the 20 fruits did not contain a Stevens allele at one or more loci. We interpreted this to mean that the fruit was from an "off genotype" which had germinated and become established in the Stevens bed. This is an example of intra-cultivar genetic heterogeneity, which is fairly common in cranberry (Fajardo et al., 2012; Novy et al., 1996).

PCA performed using the allele scores at 12 loci for the 174 Stevens seedlings revealed a single cluster of seedlings resulting from self-pollination; the remaining outcrossed seedlings were outside the cluster of self-pollinated seedlings and spread across the PCA (Fig. 2). These PCA results provided further evidence that the seedling pollen source determination based on the presence or absence of Stevens' alleles was accurate. Separation of the crosspollinated seedlings into two apparent groups by the second principal component (PC) suggests that the foreign pollen which fertilized the cross-pollinated seedlings could have come from two or more sources (Fig. 2). Additionally, the 
seedlings which came from the fruit determined to be an "off genotype" rather than a Stevens fruit fell into two distinct groups separated along the first PC. One of the groups contained other outcrossed seedlings whose mother was Stevens; and therefore, the group could represent a set of full-sib progeny from reciprocal crosses of Stevens and the "off genotype". The other group probably represents a set of progeny whose mother was the "off genotype", and which were either self-pollinated or fertilized by another foreign pollen source.

The ability of these markers to differentiate between the seedlings, even between the self-pollinated seedlings, demonstrates their potential power, precision, and accuracy for high throughput DNA fingerprinting in cranberry. Detecting the "off genotype" in the Stevens bed and the potential full-sibs from reciprocal cross of the "off genotype" and Stevens suggests that the markers can quickly and cheaply be used to assist grower's in determining the genetic purity of existing beds on their property when trueness-to-type is questioned due to observations of unique morphological differences or yield variation between beds of the same cultivar. Furthermore, the markers should allow licensed propagators to monitor the genetic homogeneity of beds used to grow vines for sales and should help breeders maintain the intellectual property rights of hybrid cultivars in cases where license agreements may be violated. Each new cultivar released can have a known genetic fingerprint based on all 61 markers from the 16 multiplexing panels, which can be used for accurate identification by researchers and personnel throughout the industry whenever necessary in the future. Finally, the use of these markers in this small validation study demonstrates their applicability and utility for cranberry researchers and breeders interested in cataloging and maintaining the purity of cranberry lines, assessing cranberry genetic diversity, and designing studies of particular commercial cranberry phenomena such as self-pollination within cranberry beds.

\subsection{The majority of the seeds present in fruit from a commercial cranberry bed are self-pollinated.}

Chi-square tests comparing the number of Stevens seedlings resulting from self-pollination versus cross pollination revealed that there were significantly more self-pollinated seedlings (146 seedlings) than outcrossed seedlings in the bed (18 seedlings) at the $p<0.0001$ level. Although cranberry growers and researchers assumed that the majority of seeds in cranberry beds were self-pollinated, this is the first quantitative evidence supporting this hypothesis. Although, the ten seedlings from the fruit from an "off genotype" were removed prior to the Chi-square tests, if intracultivar heterogeneity did exist within this Stevens bed, the number of outcrossed seedlings may have been inflated by the presence of foreign pollen generated within the bed. When cranberry beds are genetically pure, viable foreign pollen sources can only be adjacent cranberry beds planted to a different cultivar. 
The disproportionate number of self- versus cross-pollinated seeds within commercial cranberry beds, while not surprising, suggests that the potential effect of self-pollination on cranberry fruit size and yield is worth studying. Previous studies of self- and cross-pollinations in a diallel experiment with eight cranberry cultivars (Sarracino and Vorsa, 1991) and with two early selections (MacKenzie, 1995) showed that self-pollination leads to fruits with less seeds compared to fruits with cross-pollinated seeds. Studies in a closely related species to cranberry, high bush blueberry ( $V$. corymbosum), also revealed that cross-pollinations favored the development of higher number of seeds (Dogterom et al., 2015; Vander Kloet, 1991). Vander Kloet (1984) also studied the effect of the number of pollen donors in seed production and weight in highbush blueberry and showed that when flowers were pollinated with pollen of three donors, the fruit set, seed number and weight were higher than when pollen of a single donor was used. Therefore, if the same phenomena also occurs in cranberrry, current commercial cranberry cultivation practices of using established beds of a single cultivar may have negative impacts on fruit production and fruit quality. Future research studies should be conducted which study the effect of seed number and pollen source on cranberry fruit set, fruit weight, and yield to determine if cranberry growers could potentially achieve the increases in fruit size and total yield in their cranberry beds

by adopting practices which promote cross-pollination on their farms. Such practices could include a variety of strategies including planting adjacent beds in different cultivars, planting a mix of cultivars in a single bed, or choosing active pollinators which move between multiple beds in order to increase the spread of foreign pollen.

\section{Conclusions}

This study developed sixteen SSR multiplexing panels which can be used with M13 universal primers for highthroughput DNA fingerprinting in cranberry. The panels contained a total of 61 markers evenly distributed throughout

the genome, which easily separated important commercial cranberry cultivars. In addition, a subset of these multiplexing panels were used to genotype seedlings extracted from fruits in a cranberry bed planted to the cultivar Stevens. The seedlings were determined to be either self-pollinated or cross-pollinated using presence/absence of Stevens alleles combined with PCA, and chi-square tests provided the first quantitative evidence that the majority of seeds in commercial cranberries are self-pollinated. Therefore, the efficient and powerful DNA fingerprinting made possible by these multiplexing panels of SSR markers represents an important and applicable resource in the cranberry industry for assessing the purity of grower and licensed propagator cranberry vines, protecting intellectual property rights, assisting growers in determining genetic purity of existing beds, and for enabling genetic research and analysis of genetic diversity in cultivated, breeding and wild cranberry germplasm. 


\section{Acknowledgements}

J.Z. and B.S. wish to express their gratitude through Mt 10:32. This project was supported by USDA-ARS (project no. 3655-21220-001-00 provided to J.Z.); WI-DATCP (SCBG Project \#14-002); National Science Foundation (DBI-1228280); Ocean Spray Cranberries, Inc.; Wisconsin Cranberry Growers Association; Cranberry Institute. B.S. was supported by the Frank B. Koller Cranberry Fellowship for Graduate Students. The authors also thank Nicholi Vorsa, Eric Zeldin, Brent McCown, the Valley Corp., Cranberry Creek Inc., and NCGR for providing cranberry germplasm used in this study. We thank the anonymous reviewers who helped to enhance the quality of this paper.

\section{Author Contribution}

B.S., J.B-M.., and J.Z. conceived the research and designed the experiments. B.S. collected the fruits containing seedlings in the study. J.B-M. germinated and grew the seedlings. B.S. and S.H. selected primers, designed, multiplexing panels, and ran PCR reactions. B.S. and J.B-M. analyzed resulting data. B.S. and J.Z. wrote the manuscript. B.S. and J.Z. oversaw the entire study. 
Table 1. Cranberry (V accinium. macrocarpon) cultivars, breeding selections, and V. oxycoccos (diploid) accession genotyped using the 16 SSR multiplex panels.

\begin{tabular}{|c|c|c|}
\hline Cultivar & Pedigree & Accession Origin* \\
\hline BenLear & Native Selection, WI, USA & Dubay \\
\hline Bergman & Early Black x Searles & Dubay \\
\hline Demoranville & Franklin x Ben Lear & Rutgers \\
\hline Early Black & Native Selection, MA, USA & Dubay \\
\hline Franklin & Early Black x Howes & Dubay \\
\hline GH1 & McFarlin x Searles & Valley \\
\hline GH2 & McFarlin x Searles & Valley \\
\hline Howes & Native Selection, MA, USA & Dubay \\
\hline HyRed & Stevens x Ben Lear & UW \\
\hline LeMunyon & Native Selection, NJ, USA & Rutgers \\
\hline LoRed & Stevens x Ben Lear & UW \\
\hline Pilgrim & McFarlin x Prolific & Rutgers \\
\hline Potter's Favorite & Native Selection, WI, USA & Dubay \\
\hline Stevens & McFarlin x Potter's Favorite & PI-614078 \\
\hline V. oxycoccos (diploid) & Native Selection, AK, USA & Rutgers \\
\hline V07 & HyRed x GH1 & UW \\
\hline V11 & HyRed x LoRed & UW \\
\hline Wilcox & Howes x Searles & Dubay \\
\hline Yellow Bell & Native Selection, ME, USA & PI-555028 \\
\hline
\end{tabular}

*Dubay Cranberries Co., Junction City, WI; PE Marucci Center, New Jersey Agricultural Station, Rutgers University Chatsworth, NJ; University of Wisconsin-Madison; National Clonal Germplasm Repository, Oregon. 
Table 2. Cranberry (Vaccinium macrocarpon) PCR multiplexing panels containing SSR markers with non-overlapping allele ranges. Primer names, NCBI ID, publication origin, and position within the 12 cranberry linkage groups (LGs)

(Schlautman et al., 2015b) and allele range (bp) are reported. The subset of multiplexing panels used to genotype selfand cross-pollinated seedlings from the cultivar, Stevens, are indicated.

\begin{tabular}{|c|c|c|c|c|c|}
\hline Multiplexing panel & Primer Name & NCBI ID & LG & Position & Allele range (bp) \\
\hline \multirow[t]{3}{*}{ Panel 1} & $\operatorname{ct95345^{a}}$ & KР279106 & 7 & 30.93 & $117-128$ \\
\hline & SCF124322 ${ }^{a}$ & KP278849 & 1 & 77.97 & $213-237$ \\
\hline & SCF149976 ${ }^{a}$ & KР278893 & 5 & 51.26 & $273-317$ \\
\hline \multirow[t]{3}{*}{ Panel 2} & SCF136207 ${ }^{a}$ & KP278866 & 9 & 59.27 & $161-184$ \\
\hline & $\operatorname{ct} 155339^{a}$ & KP279140 & 3 & 27.31 & $221-230$ \\
\hline & $\operatorname{vm} 52682^{b}$ & JF834282 & 4 & 36.88 & $271-292$ \\
\hline \multirow[t]{4}{*}{ Panel 3T } & $\mathrm{SCF} 213102^{a}$ & KР278953 & 2 & 37.12 & $149-159$ \\
\hline & SCF71184 ${ }^{a}$ & KP278754 & 8 & 46.00 & $197-233$ \\
\hline & SCF92414 ${ }^{a}$ & KP278793 & 6 & 34.93 & $285-289$ \\
\hline & 409500_K63 ${ }^{a}$ & KP279177 & 10 & 53.03 & $333-393$ \\
\hline \multirow[t]{4}{*}{ Panel 4} & $\operatorname{scf} 9 e^{c}$ & $\mathrm{~N} / \mathrm{A}$ & 5 & 67.28 & $184-206$ \\
\hline & $\mathrm{SCF} 149633^{a}$ & KР278891 & 1 & 24.33 & $292-325$ \\
\hline & $\operatorname{scf} 4860^{\circ}$ & $\mathrm{N} / \mathrm{A}$ & 8 & 34.85 & $349-398$ \\
\hline & $\operatorname{scf} 439^{\circ}$ & $\mathrm{N} / \mathrm{A}$ & 9 & 36.58 & $513-524$ \\
\hline \multirow[t]{4}{*}{ Panel 5} & $\operatorname{ct89711^{a}}$ & KР279102 & 3 & 22.42 & $137-150$ \\
\hline & SCF110888 ${ }^{a}$ & KР278826 & 3 & 70.05 & $179-193$ \\
\hline & SCF118999 ${ }^{a}$ & KР278842 & 1 & 78.98 & $270-309$ \\
\hline & SCF72229 & KР278756 & 4 & 30.61 & $322-345$ \\
\hline \multirow[t]{4}{*}{ Panel 6} & 1 trimcontig $344502^{a}$ & KР279241 & 6 & 56.80 & $133-164$ \\
\hline & SCF104688 ${ }^{a}$ & KР278809 & 10 & 67.07 & $200-223$ \\
\hline & SCF138014 $4^{a}$ & KР278870 & 7 & 37.67 & $246-248$ \\
\hline & SCF21596 ${ }^{a}$ & KP278655 & 11 & 41.89 & $303-323$ \\
\hline \multirow[t]{3}{*}{ Panel 7} & $\operatorname{scf3} 2 j^{c}$ & $\mathrm{~N} / \mathrm{A}$ & 9 & 82.53 & $132-154$ \\
\hline & $\mathrm{SCF} 138394^{a}$ & KP278871 & 12 & 29.72 & $217-244$ \\
\hline & $\mathrm{SCF} 82870^{a}$ & KP278775 & 8 & 35.70 & $276-313$ \\
\hline
\end{tabular}




\begin{tabular}{|c|c|c|c|c|c|}
\hline & 29080_K63a & KP279160 & 2 & 71.39 & 383-398 \\
\hline \multirow[t]{3}{*}{ Panel 8} & SCF132595 & KP278863 & 5 & 27.67 & $227-281$ \\
\hline & $\operatorname{scf} 35 k^{c}$ & $\mathrm{~N} / \mathrm{A}$ & 9 & 41.03 & $297-315$ \\
\hline & 309084_K70 & KP279212 & 12 & 40.33 & $389-401$ \\
\hline \multirow[t]{4}{*}{ Panel 9† } & SCF140628 ${ }^{a}$ & KP278876 & 4 & 33.90 & 144-169 \\
\hline & SCF59248 & KP278740 & 1 & 52.75 & 194-205 \\
\hline & SCF107715 ${ }^{a}$ & KP278816 & 10 & 39.34 & $247-304$ \\
\hline & SCF73288 & KP278758 & 2 & 65.01 & $320-334$ \\
\hline \multirow[t]{4}{*}{ Panel 10} & 1trimcontig217158 & KP279229 & 7 & 52.21 & $154-174$ \\
\hline & SCF $27811^{a}$ & KP278672 & 8 & 48.08 & $220-262$ \\
\hline & SCF31208 ${ }^{a}$ & KP278687 & 5 & 25.81 & $306-361$ \\
\hline & 121633_K63a & KP279163 & 3 & 76.73 & $400-426$ \\
\hline \multirow[t]{4}{*}{ Panel 11} & $\operatorname{ct} 154615^{a}$ & KP279138 & 6 & 20.13 & $131-140$ \\
\hline & VCC_J9 $9^{d}$ & AY762683 & 9 & 23.36 & $171-220$ \\
\hline & SCF38942 ${ }^{a}$ & KP278704 & 12 & 31.07 & $233-262$ \\
\hline & SCF73288 & KP278758 & 2 & 65.01 & $289-333$ \\
\hline \multirow[t]{4}{*}{ Panel 12} & $\operatorname{vm} 68798^{b}$ & JF834242 & 11 & 47.89 & $152-170$ \\
\hline & $\operatorname{vm} 25796^{b}$ & JF834263 & 10 & 52.83 & $197-267$ \\
\hline & SCF85773 & KP278780 & 3 & 34.09 & $285-304$ \\
\hline & $\mathrm{SCF} 18709^{a}$ & KP278651 & 2 & 50.35 & $344-354$ \\
\hline \multirow[t]{4}{*}{ Panel 13} & $\operatorname{vm} 72062^{b}$ & JF834244 & 10 & 41.93 & $147-170$ \\
\hline & SCF20681 ${ }^{a}$ & KP278653 & 7 & 76.07 & $220-236$ \\
\hline & SCF $3551^{a}$ & KP278608 & 1 & 93.53 & $264-290$ \\
\hline & 297265_K63 & KP279167 & 2 & 48.67 & $331-343$ \\
\hline \multirow[t]{4}{*}{ Panel 14† } & SCF113389 ${ }^{a}$ & KP278831 & 4 & 31.54 & $150-169$ \\
\hline & 314797_K70 & KP279218 & 8 & 48.58 & $202-224$ \\
\hline & $\operatorname{vm} 27120^{b}$ & JF834265 & 6 & 41.90 & $255-269$ \\
\hline & 308539_K70 & KP279209 & 11 & 54.52 & $305-315$ \\
\hline
\end{tabular}




\begin{tabular}{|c|c|c|c|c|c|}
\hline \multirow[t]{4}{*}{ Panel 15} & SCF8987 ${ }^{a}$ & KP278625 & 5 & 51.16 & $151-157$ \\
\hline & 372875_K63 ${ }^{a}$ & KP279174 & 2 & 66.03 & $198-230$ \\
\hline & SCF37628 ${ }^{a}$ & KP278700 & 12 & 43.49 & $258-267$ \\
\hline & SCF53750 ia & KP278727 & 3 & 61.68 & $315-352$ \\
\hline \multirow[t]{4}{*}{ Panel 16} & SCF7155 ${ }^{a}$ & KP278617 & 7 & 73.80 & $169-198$ \\
\hline & SCF97378 ${ }^{a}$ & KP278801 & 5 & 32.84 & $238-254$ \\
\hline & SCF88396 ${ }^{a}$ & KР278784 & 9 & 62.36 & $287-296$ \\
\hline & SCF59739 $9^{a}$ & KP278741 & 12 & 53.07 & $336-352$ \\
\hline
\end{tabular}

T Panel used to genotype self- and cross-pollinated seedlings from the cultivar, Stevens.

${ }^{a}$ Published in Schlautman et al., 2015a.

${ }^{b}$ Published in Zhu et al., 2012.

'Published in Georgi et al., 2013.

${ }^{d}$ Published in Boches, 2005. 


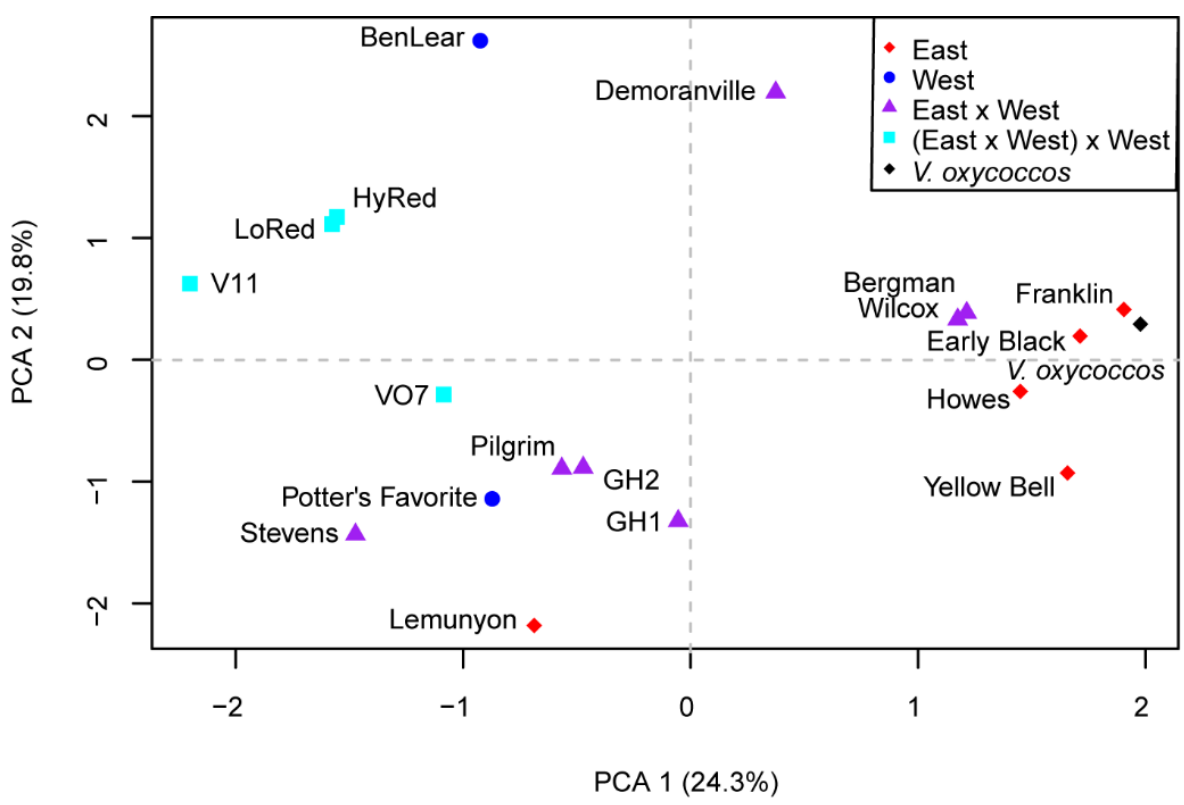

Fig 1. Principal component analysis for 18 cranberry (V accinium macrocarpon) cultivars, breeding selections, and $V$. oxycoccos accession based on genotypic data from 61 short sequence repeats (SSRs) multiplexed in 16 panels. Genotypes are plotted and colored based on their selection origin (either east or west of the Appalachian Mountains) or the selection origin within a specific cultivar's pedigree (i.e. either [east x west] or [[east x west] x west]). 


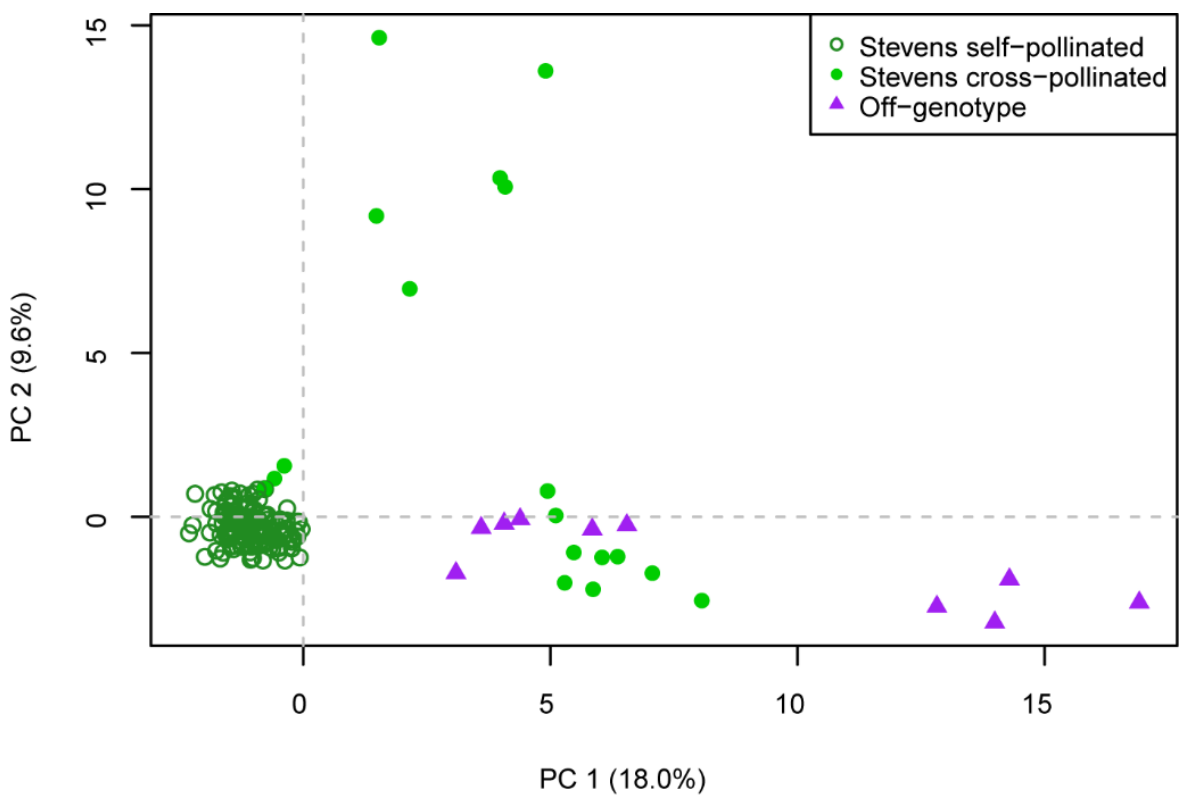

Fig 2. Principal component analysis of 174 cranberry (V accinium macrocarpon) seedlings extracted from 20 fruits collected in a commercial bed of the cultivar Stevens. Seedlings were determined to be either self-pollinated, cross-pollinated by a foreign pollen source, or had a mother which was an "off-genotype" which had somehow established itself in the Stevens bed. 


\section{References}

Boches, P., (2005) Microsatellite marker development and molecular characterization in Highbush Blueberry (Vaccinium corymbosum L.) and Vaccinium species. M.S. Diss., Oregon State Univ., Corvallis, OR.

Brown, A., McNeil, J., (2006) Fruit production in cranberry (Ericaceae: Vacinium macrocarpon): A bet-hedging strategy to optimize reproductive effort. Am. J. Bot. 93, 910-916.

Brownstein, M. J., Carpten, J. D., Smith, J. R., (1996) Modulation of non-templated nucleotide addition by Taq DNA polymerase: primer modifications that facilitate genotyping. Biotechniques, 20, 1004-6, 1008-10.

Chandler, F., Demoranville, I., (1958) Cranberry varieties of North American, 513th ed. Massachusetts Agricultural Experimental Station Bulletin.

Clark, J.R., Finn, C.E., (eds.). (2010) Register of new fruit and nut cultivars, list 45. HortSci., 45, 716-756.

Dana, M., (1983) Cranberry cultivar list. Frt. Var. J. 37, 88-95.

Dogterom, M.H., Winston, M.L., Mukai, A.M.Y. (2015) Effect of pollen load size and source (self, outcross) on seed and fruit production in highbush blueberry cv. 'Bluecrop' (V accinium corymbosum; Ericaceae). Am. J. Bot., 87, 15841591.

Dray, S., Dufour, A.B., (2007) The ade4 Package: Implementing the Duality Diagram for Ecologists. J. Stat. Softw., 22, 1 -20 .

Fajardo, D., Morales, J., Zhu, H., Steffan, S.A., Harbut, R., Bassil, N., Zalapa, J., (2012) Discrimination of American cranberry cultivars and assessment of clonal heterogeneity using microsatellite markers. Plant Mol. Biol. Rep. 31, 264-271.

Georgi, L., Herai, R.H., Vidal, R., Carazzolle, M.F., Pereira, G.G., Polashock, J., Vorsa, N., (2011) Cranberry microsatellite marker development from assembled next-generation genomic sequence. Mol. Breed., 30, 227237.

Georgi, L., Johnson-Cicalese, J., Honig, J., Das, S.P., Rajah, V.D., Bhattacharya, D., Bassil, N., Rowland, L.J., Polashock, J., Vorsa, N., (2013) The first genetic map of the American cranberry: exploration of synteny conservation and quantitative trait loci. Theor. Appl. Genet., 126, 673-692.

Guichoux, E., Lagache, L., Wagner, S., Chaumeil, P., Léger, P., Lepais, O., Lepoittevin, C., Malausa, T., Revardel, E., Salin, F., Petit, R.J., (2011) Current trends in microsatellite genotyping. Mol. Ecol. Resour., 11, 591-611.

McCown, B.H., Zeldin, E.L., (2003) 'HyRed', an early, high fruit color cranberry hybrid. HortSci., 38, $304-305$.

Mackenzie, K., (1995) Pollination requirements of the American cranberry. J. Small Fruit Vitic., 2, 33-44.

Nilsen, E.T., (1995) Phenotypic Plasticity and genetic variation of V accinium macrocarpon, the American cranberry. I. Reaction norms of clones from central and marginal populations in a common garden. Int. J. Plant Sci., 156, 687.

Novy, R., Vorsa, N., (1995) Identification of intracultivar genetic heterogeneity in cranberry using silver-stained RAPDs. HortSci., 30, 600-60.

Novy, R.G., Vorsa, N., Patten, K., (1996) Identifying genotypic heterogeneity in 'McFarlin' cranberry: A randomlyamplified polymorphic DNA ( RAPD ) and phenotypic analysis. J. Amer. Soc. Hort. Sci., 121, $210-215$.

Peltier, G., (1970) A history of the cranberry industry in Wisconsin. Detroit, Michigan: Harlo Press.

Polashock, J., Vorsa, N., (2002) Development of SCAR markers for DNA fingerprinting and germplasm analysis of American cranberry. J. Amer. Soc. Hort. Sci., 127, 677-684.

Sarracino J. M., Vorsa, N., (1991) Self and cross fertility in cranberry. Euphytica, 58, 129-136. 
Schlautman, B., Fajardo, D., Bougie, T., Wiesman, E., Polashock, J., Vorsa, N., Steffan, S., Zalapa, J., (2015a) Development and validation of 697 novel polymorphic genomic and EST-SSR markers in the American cranberry (Vaccinium macrocarpon Ait.). Molecules, 20, 2001-2013.

Schlautman, B., Covarrubias-Pazaran, G., Diaz-Garcia, L.A., Johnson-Cicalese, J., Iorrizo, M., Rodriguez-Bonilla, L., Bougie, T., Bougie, T., Wiesman, E., Steffan, S., Polashock, J., Vorsa, N., Zalapa, J., (2015b) Development of a high-density cranberry SSR linkage map for comparative genetic analysis and trait detection. Mol. Breed., 35, 177.

Schuelke, M., (2000) An economic method for the fluorescent labeling of PCR fragments A poor man's approach to genotyping for research and high-throughput diagnostics. Nat. Biotechnol., 18, 1-2.

Smith, T. W., Walinga, C., Wang, S., Kron, P., Suda, J., Zalapa, J., Evaluating the relationship between diploid and tetraploid Vaccinium oxycoccos L. (Ericaceae) in eastern Canada. Botany, 93, 623-636.

Staub J.E., and Meglic, V., (1993) Molecular genetic markers and cultivar discrimination: a case study in cucumber. Horttechnology, 3, 291-300.

Vander Kloet, S., (1984) Effect of pollen donors on seed production, seed weight, germination and seedling vigor in Vaccinium corymbosum L. Am. Midl. Nat., 112, 392-396.

Vander Kloet, S.P., (1991) The consequences of mixed pollination on seed set in Vaccinium corymbosum. Can. J. Bot., 69, 2448-2454.

Vorsa, N., Polashock, J., Alteration of anthocyanin glycosylation in cranberry through interspecific hybridization. J. Amer. Soc. Hort. Sci., 130, 711-715.

Zalapa, J.E., Bougie, T.C., Bougie, T.A., Schlautman, B.J., Wiesman, E., Guzman, A., Fajardo, D.A., Steffan, S., Smith., T., (2014) Clonal diversity and genetic differentiation revealed by SSR markers in wild $V$ accinium macrocarpon and Vaccinium oxycoccos. Ann. Appl. Biol., 166, 196-207.

Zhu, H., Senalik, D., McCown, B.H., Zeldin, E.L., Speers, J. Hyman, J., Bassil, N., Hummer, K., Simon, P.W., Zalapa, J.E., (2012) Mining and validation of pyrosequenced simple sequence repeats (SSRs) from American cranberry (V accinium macrocarpon Ait.). Theor. Appl. Genet., 124, 87-96. 